\title{
A STRATEGIC PLANNER FOR ROBOT EXCAVATION ${ }^{1}$
}

\author{
by \\ Humberto Romero-Lois, Research Assistant, \\ Department of Civil Engineering \\ Chris Hendrickson, Professor, \\ Department of Civil Engineering, and \\ Irving Oppenheim, Associate Professor \\ Departments of Architecture and Civil Engineering \\ Camegie Mellon University \\ Pittsburgh, PA 15213-3890 USA
}

\begin{abstract}
This paper describes the implementation of a domain-specific strategic planner for an autonomous excavator, called EXCAVATION PLANEX. As a knowledge-based expert system, the planner is designed to assist in the automation of a power-shovel robotic excavator that performs open-cut excavation. The system has been applied in laboratory simulations independently as an automated planner. It plans for the excavation of a uniform sloping terrain given a desired excavation layout and the conditions observed during the actual process. Partitioning the site into excavation sectors, defining reachable sections, and formulating basic strategic actions to perform (e.g. Move Excavator, Dig Section and Scan Surroundings) are part of the system tasks. The planner uses a generic knowledge-based system architecture for process planning, PLANEX, which has been used in various application domains. This paper includes a description of the architecture, the control procedures and the knowledge representation.
\end{abstract}

\section{INTRODUCTION}

Excavation is a process in which two fundamental objects interact: an external actor and the environment itself. The terrain characteristics and features, which define an unstructured environment, are modified in the process by the external actor, "the excavator". The excavation's goal and state, as well as the excavator, can be described adequately by computer models. These conditions and the overall economic importance of excavation motivate the goal of having a robot excavator that would be given the location and dimensions of a desired excavation, and would plan the excavation process, sense the environment, react to changes as excavation proceeds, and accomplish the desired excavation with almost no external or human intervention. Such a system is known as a "cognitive robot" [Whittaker 85].

Cognitive robots require information for modeling, planning and acting at different levels of abstraction, corresponding to plans, tasks, commands, and external representations that are decomposed into various levels of detail. This hierarchy aides in a fast reflexive response and for assuring at the same time the robot's strategic performance and long term mission accomplishment. In order to coordinate the robot actions with the surroundings, the planning system has to predict and dynamically plan for future situations (at different levels of detail) while acting in the present.

The implementation of a strategic planning system for a robotic excavator was motivated by its importance in the development of a complete autonomous machine that can accomplish its mission in a dynamic environment. The need of a strategic-dynamic planner is clear if the complete excavation process is to be handled autonomously by the excavator. Various issues in the development of the software structure of an autonomous robotic excavator have been studied, giving more emphasis to the tactical and reflexive levels [Tatsuro 88, Bullock 88, Bullock 89]. The system described here, EXCAVATION PLANEX, is intended to be part of this research, studying the role of the planning and monitoring systems of a cognitive robot, and summarizing a set of characteristics and elements to consider in the automation of excavation at the strategic level.

\footnotetext{
${ }^{1}$ Prepared for the Sixth International Symposium on Automation and Robotics in Construction, San Francisco, CA, June 1989.
} 
EXCAVATION PLANEX consists of a strategic planning and monitoring system for a front shovel robotic excavator [Romero 88]. The planning functions include synthesizing primitive action sequences into plans that achieve the desired objectives, and controlling and monitoring the plan execution. Once a sequence of actions is generated, the execution of each action alters the environment in a way that may need a re-evaluation of the already obtained sequence of actions. The planner should interact at a lower level with a tactical planner whose work is based on local information. It assumes the use of sensors for the acquisition and measurement of range data used to update the strategic model of the environment. The model is a strategic test frame since different lower level planners that handle local geometry and robot physics may be developed for this domain-specific case.

In the excavation problem, strategic plans require the generation and ordering of a sequence of excavation sections or volumes to excavate, the definition of the gross vehicle movements and positioning, and the estimation of the required time of completion of the excavation. These tasks are based on a global model of the excavation site and take into account the desired excavation to accomplish, a priori knowledge about the domain such as the machine dimensions and working ranges, and the terrain characteristics. The strategic planner does not concern itself with how the excavating tool accomplishes work within each volume and how the machine moves from one position to another. This is assumed to be consider by a lower level planner, more robust so that it may perform hit detection, assure digging operations, and determine excavating tool motions and torques. Figure 1 illustrates the strategic and tactical frames of reference. Regarding the path planning process for achieving movements from one excavation section to the other, a robot path planning and obstacle avoidance algorithm is considered necessary at the tactical level.

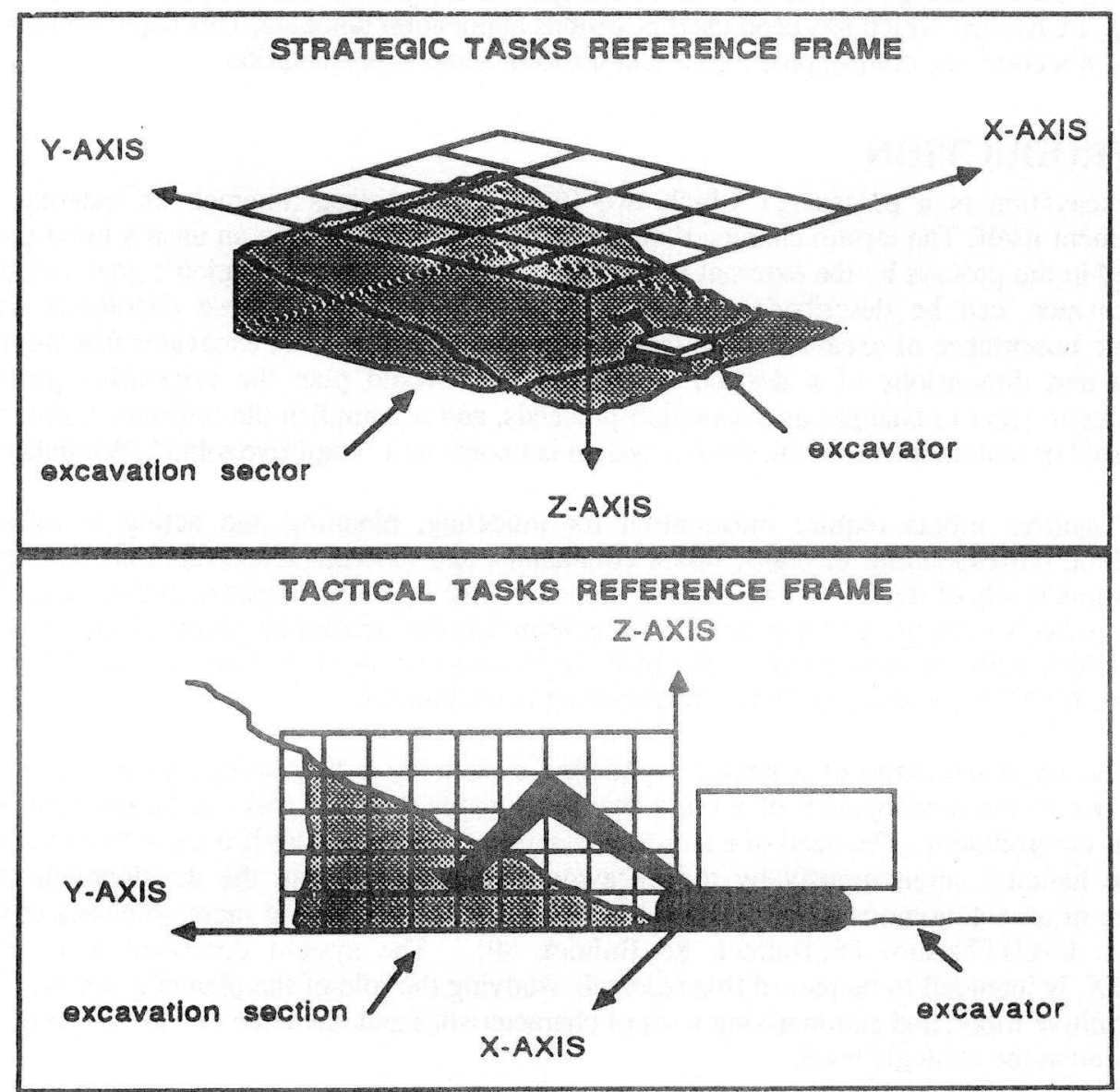

Figure 1: Strategic and Tactical Reference Frames 


\section{OVERVIEW OF EXCAVATION PLANEX}

EXCAVATION PLANEX consists of a strategic motion planner, a strategic monitor, and various interface utilities. The system was implemented using the basic components of the PLANEX architecture [Zozaya 89,Zozaya 88]: hierarchical representation structures, problem solving operators, knowledge sources, and user interface mechanisms. The system was implemented on a TI EXPLORER ${ }^{\text {TM }}$ in KNOWLEDGECRAFT ${ }^{\mathrm{TM}}$, but could be ported to the hardware independent, COMMON LISP version of PLANEX. The overall operation and data flow-chart is shown in Figure 2.

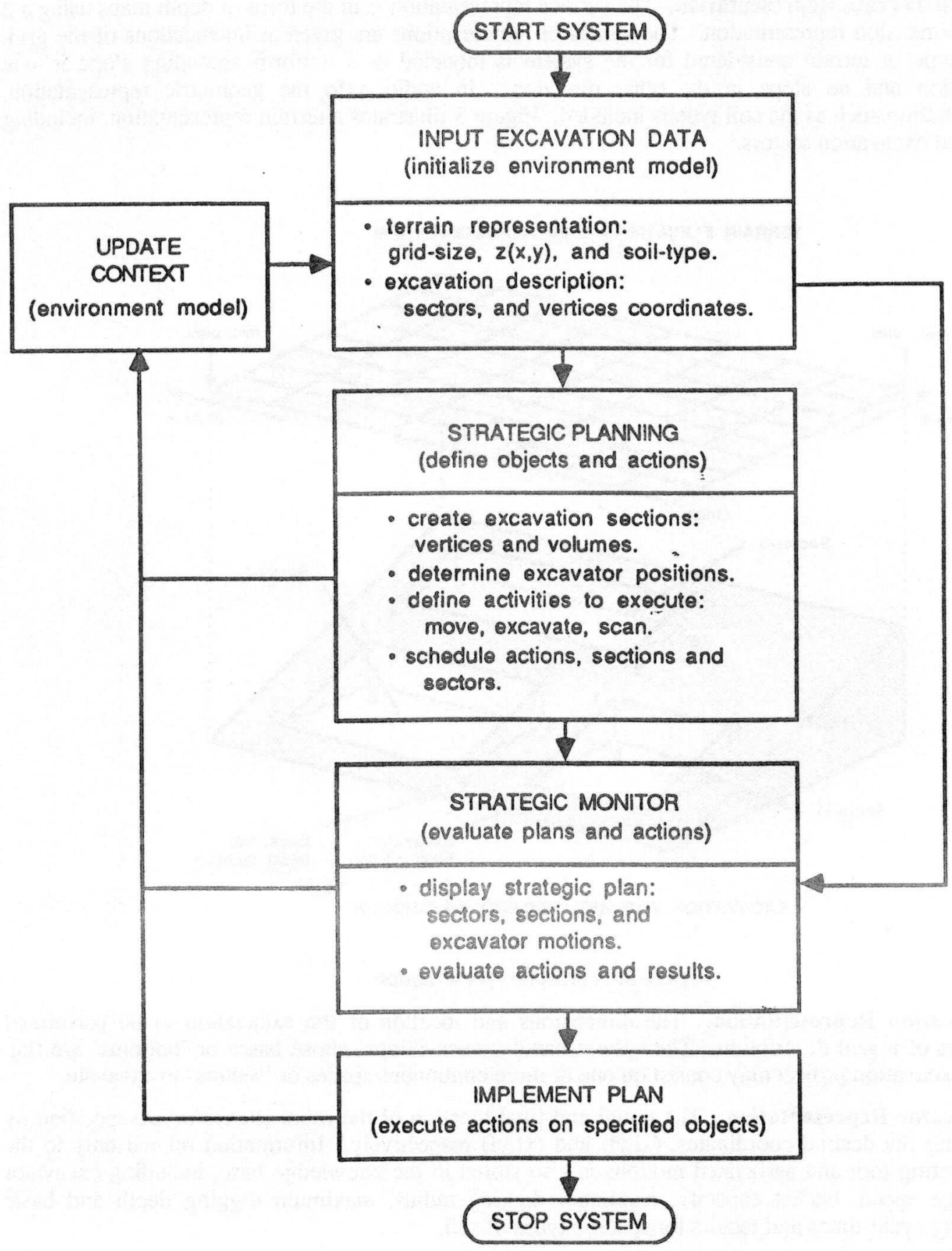

Figure 2: System operation and data flow 


\section{EXCAVATION PLANEX REPRESENTATION STRUCTURES}

The model of the environment includes three basic objects: (1) the terrain surface, (2) the description of the desired excavation, and (3) the excavator itself. The information is stored in a series of hierarchical schemas or frames. This facilitates the manipulation of data at different levels of abstraction and allows the inheritance of information at different levels of detail. The schemas are not only created as the data is entered; during the planning process, additional schemas are created to describe and represent actions and objects. The following are the basic groups of schemas identified in the system:

1. Actual Terrain Representation. The surface representation is in the form of depth maps using a 2 1/2-Dimension representation. The values of the elevations are given at intersections of the grid. The type of terrain considered for the system is modeled as a uniform ascending slope in one direction and no slope in the other direction. In addition to the geometric representation, information such as the soil type is included. Figure 3 illustrates a terrain representation, including desired excavation sectors.

TERAAN ELEVATIONS OAIO REPRESENTATION

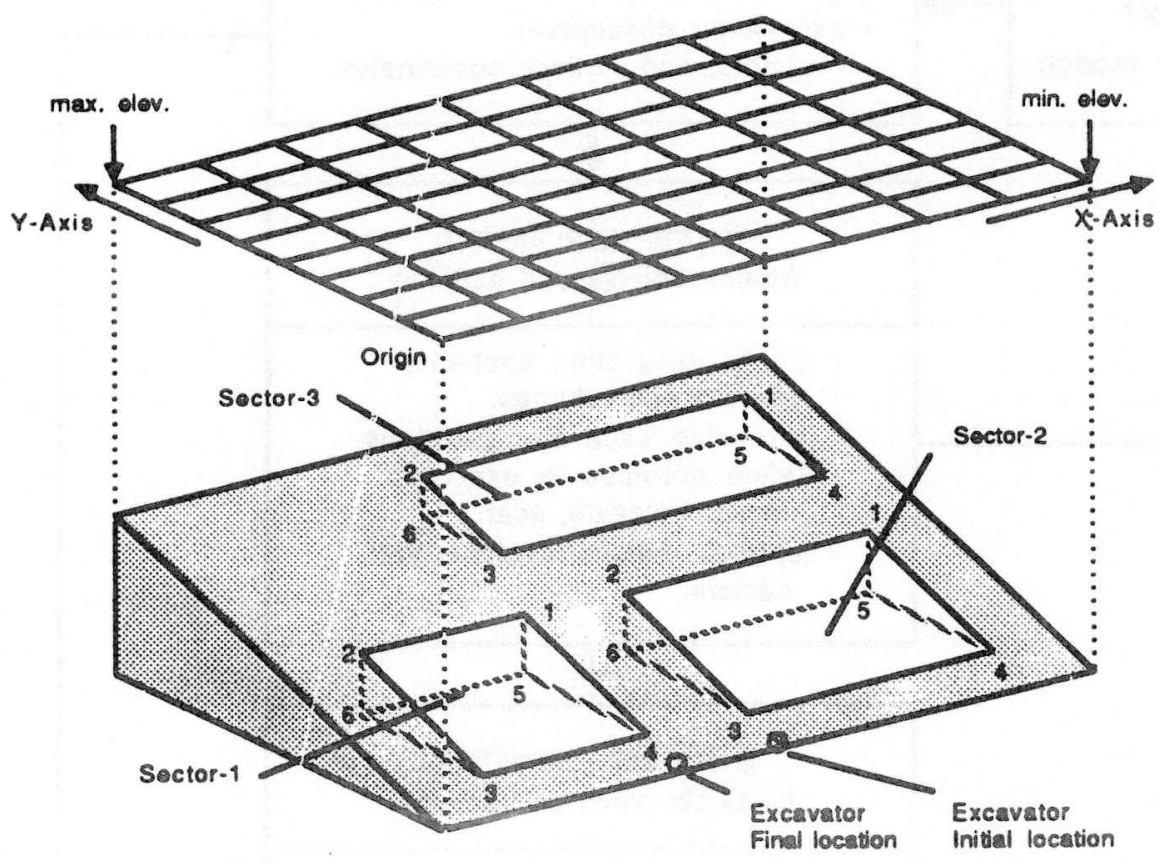

EXCAVATHON \&TE AND PMOJECT DEBCAPTION

Figure 3: Terrain Representation

2. Excavation Representation. The dimensions and location of the excavation to be performed consist of a grid description. The system handles excavations whose bases or 'bottoms' are flat. The excavation project may consist on one or more continuous spaces or 'sectors' to excavate.

3. Excavator Representation. The initial and final location of the robot excavator are specified by entering the desired coordinates, (xi,yi) and (xf,yf) respectively. Information related only to the excavating tool and associated motions is also stored in the knowledge base, including excavator average speed, bucket capacity, maximum digging radius, maximum digging depth and basic digging cycle-times and factors for specific types of soil. 


\subsection{EXCAVATION PLANEX OPERATORS}

The strategic planner defines gross excavator motions (distances to move), creates a network of positioning-digging-scanning actions, and estimates the durations and volumes of the excavations. Excavation actions must be decomposed both spatially and temporally (Figure 4). Three main actions, which are related to the excavation process only, are identified and are the input for the next lower level planning systems:

- Move-to-section: Includes the coordinates of the new location, the distance to travel (in a straight line), and the time required for this task.

- Excavate-section: The dimensions and volume of the section to excavate is estimated, as well as the time needed for this process.

- Scan-section: Gives the 'width' of the scanning path that the range sensors have to follow for updating the environment model, and the time consumed.

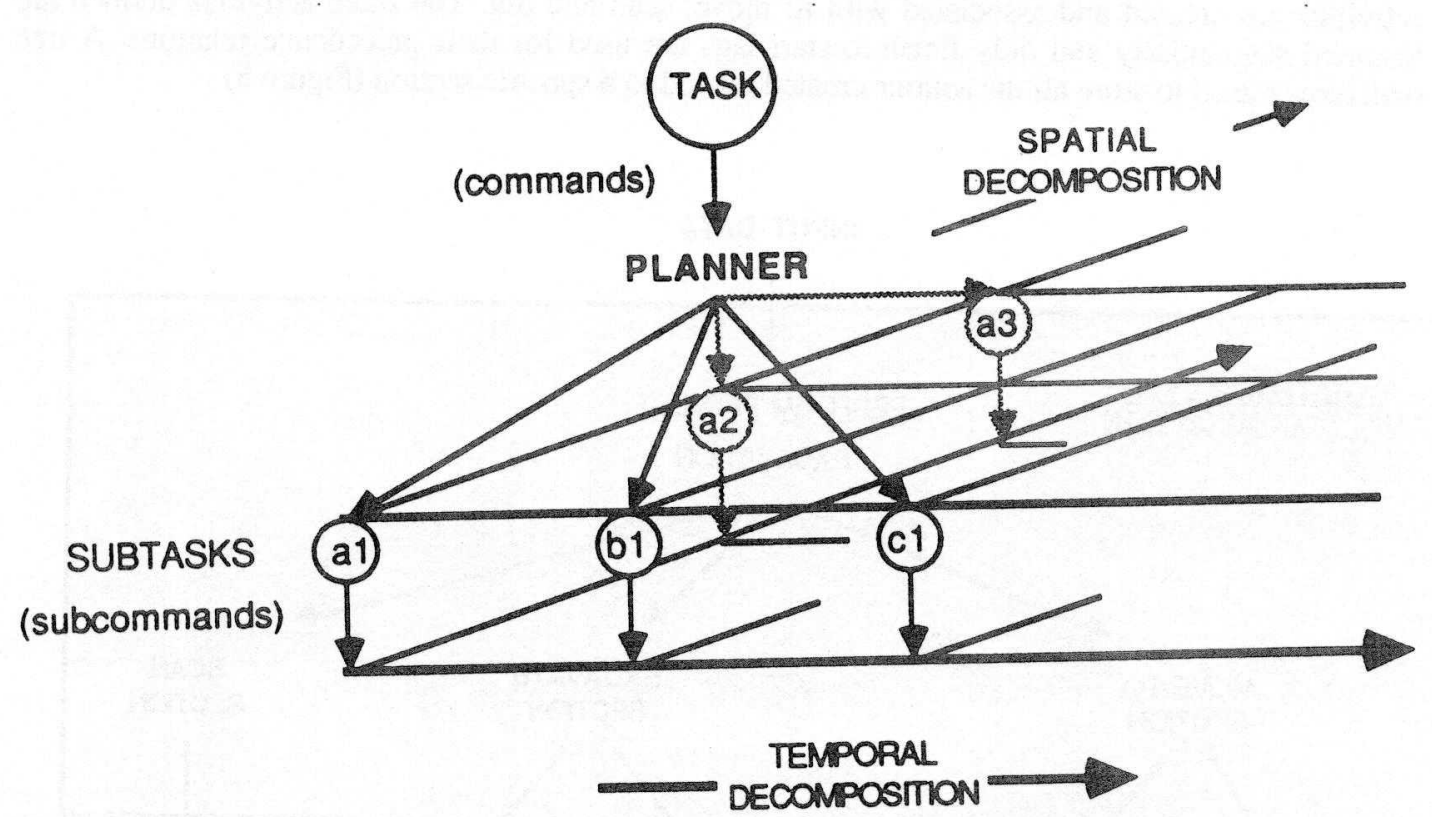

Figure 4: Decomposition of Tasks

The Excavation Strategy consists of a series of problem-solving operators that are activated by the conditions represented in the main objects and the rules stored in the knowledge sources. Each operator is a procedural function that modifies the context or data base, by creating, modifying or deleting objects.

The following are the operators, with the associated knowledge sources (representing rules and calculation functions), that the system includes:

1. Initialize the terrain representation model. The excavation strategy presumes a state model of a user-defined volume representing the site. In order to perform a reactive planning process, the system must always maintain a model of the excavation site and store all pertinent information known about the project. The desired excavation project is provided to the strategic planner as a goal representation which is initialized and updated before the robot starts working, and from which the robot extracts the information needed to generate the actions to achieve the goal-excavation.

This operator assigns the elevations of the terrain at all grid intersections, based on the input data. These elevations in the terrain grid-matrix are modified and updated with new elevations reached when a new section is excavated. 
2. Order sectors for excavation. The excavation project consists one or more continuous sectors to be excavated. This operator computes the distances from the excavator initial and final locations to the sectors vertices and designates the order in which sectors are to be excavated. This operator guarantees that any sector to be excavated will be reached as the excavators moves towards its final destination.

3. Create excavation sections and tree of sections. These operators are in charge of partitioning each excavation sector into sections which are volumes of soil reachable by the excavator from a defined position, and creating a tree of these sections schemas associated with the sector and used to define the precedence relations. The partition objective is to insure continuous excavation and minimal base motion, as well as the feasibility of the complete excavation. A Cartesian representation is used in this analysis, with limits on size provided by characteristics of the excavator and the size of the desired excavation sectors. A polar (fan-shaped) decomposition could also be used [Apte 89].

4. Create robot strategic actions and tree of actions. For each section created, the following activities are created and associated with it: move, scan and dig. The three activities defined are assumed sequentially and only finish-to-start lags are used for their precedence relations. A tree structure is used to store all the actions created related to a specific section (Figure 5).

INPUT DATA

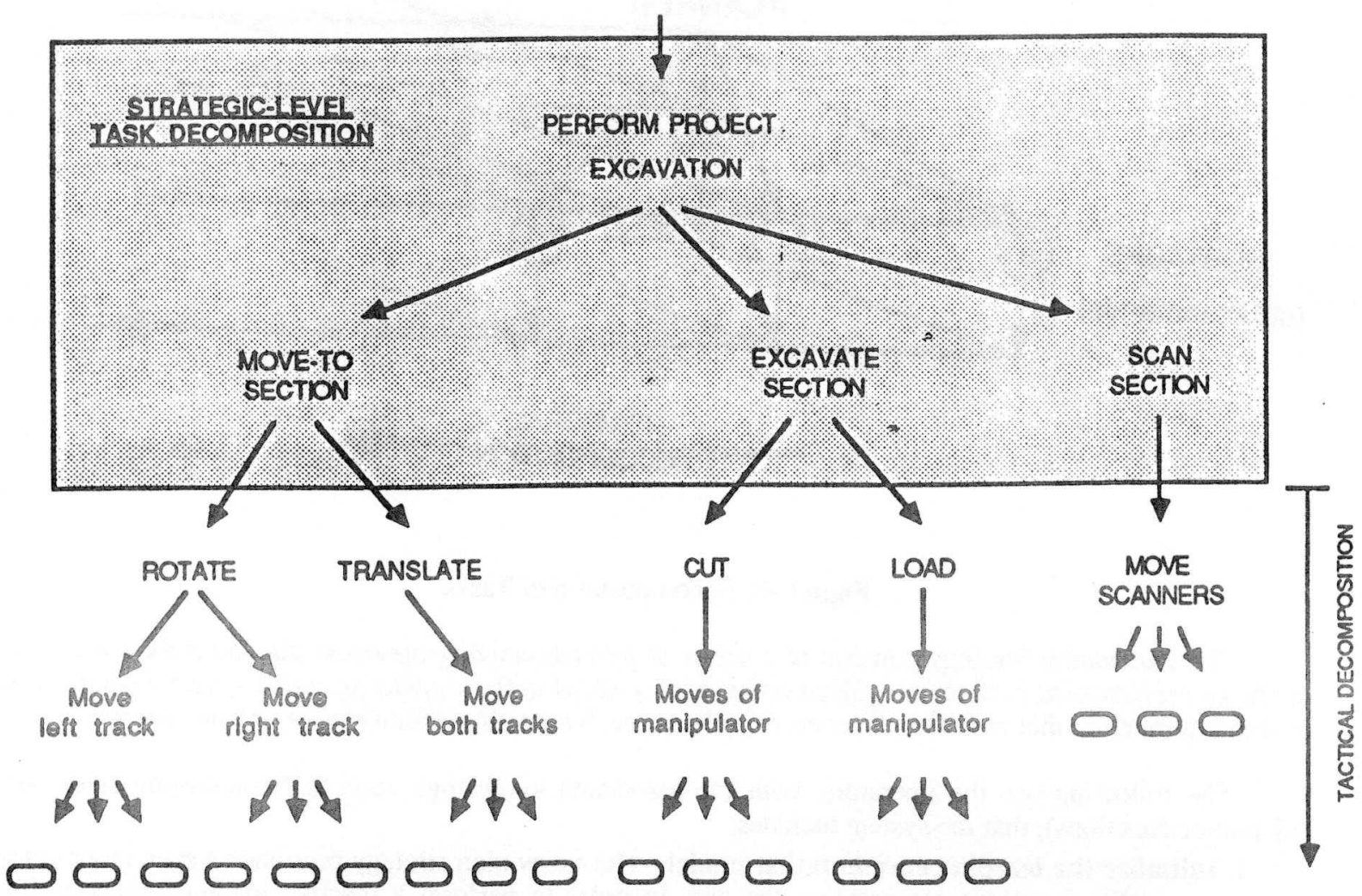

Figure 5: Actions Decomposition

5. Determine amounts of work and durations. The amount of work and time required for each action are calculated, depending on the activity type. For the moving actions, the amount of work is equal to the distance from previous position to the next position in the new section. For the digging action this is equal to the volume of soil to remove from the section. Finally for the scanning action this is the distance of the symbolic path that the sensors have to follow. The durations are obtained 
considering the different cycle times and factors defined by the system for the given conditions.

The total duration of the excavation project is obtained by the durations operator, as well as the early start time and early finish time at which the actions in each section will take place. This is done using a simple 'Critical Path Method' algorithm [Hendrickson 89].

6. Simulate the excavation process and monitor the plan. The simulation of the plan developed is done using a display format. Once a plan is generated and the excavator starts operating, the display shows the robot positions and operations as they are performed. The simulation asks the user to enter a given condition that may differ from the expected result obtained by the plan, or be within an acceptable margin. The planner then evaluates the result and if necessary repeats the complete planning process for the unfinished excavation. When the complete plan is executed, both the actual and goal representations have to be equal so that the system may stop operating. Figure 6 illustrates the graphic simulation.

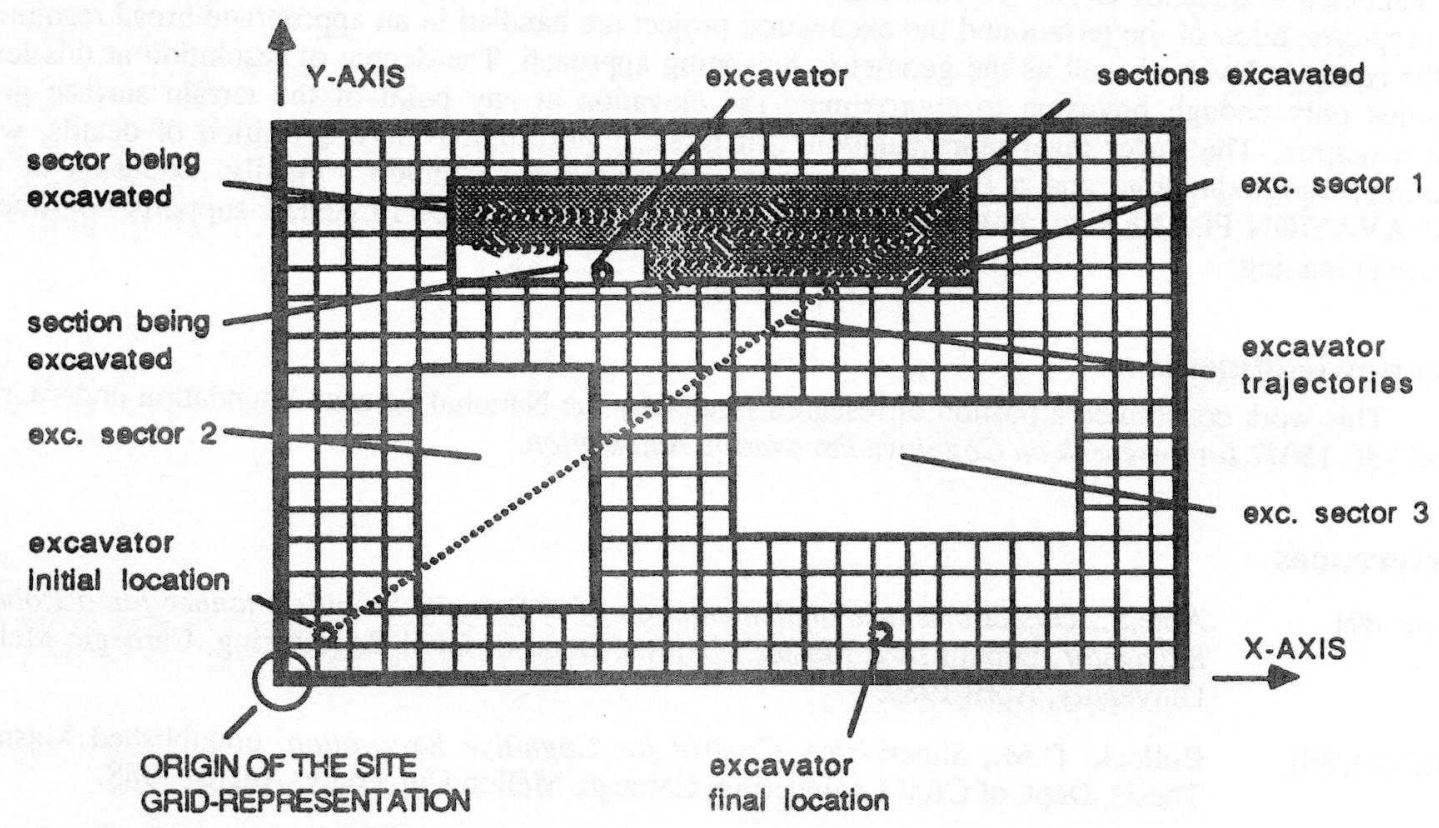

Figure 6: Excavation Plan Monitoring Display

\section{EXCAVATION PLANEX KNOWLEDGE SOURCES}

The knowledge required by the operators is stored in groups of rules called knowledge sources. Each knowledge source provides information for a particular operation such as sections creation, actions formulation, duration estimation or precedence determination.

The types of knowledge sources included in the system are:

- Amount Knowledge Sources: These indicate the formulas for computing the amount of work for each action (excavate, move and scan), as defined by its associated operator.

- Duration Knowledge Sources: These are used for estimating the digging-moving cycle time for the given conditions, and compute the durations of the actions previously defined.

- Successor Knowledge Sources: These obtain the precedence relations for the actions considered, indicating the successors for each action. For the present application, the successors are identified by the activity code.

In addition to these knowledge sources, domain independent knowledge sources used for creating the trees of excavation sections and actions were adapted directly from the PLANEX architecture. 
The various knowledge sources use stored information concerning the terrain, the excavator itself, and the goals and current state of the plan. Knowledge sources can also invoke computational or algorithmic procedures.

\section{SUMMARY AND CONCLUSIONS.}

The present work covered mainly elements related to autonomous planning in an excavation domain, to investigate the feasibility and conditions of such a project at a strategic level of abstraction. The generation of 'long term' or strategic plans and its execution monitoring, were sufficiently accomplished in the present system. During the system development and implementation, it became apparent that the lower levels of a cognitive robot architecture require more attention and knowledge. Although the strategic planning and monitoring process is required for a complete automated excavation process, its final implementation depends largely on the results of the research done at the tactical and operational levels. The representation of the terrain and the excavation project are handled in an appropriate broad resolution at the strategic level, as well as the geometric reasoning approach. The degree of resolution at this level requires only enough precision to approximate the elevation at any point of the terrain surface gridrepresentation. The lower level representation structures, require much more definition of details, with minimum approximation, and a fine environment representation resolution. Finally, extension of the EXCAVATION PLANEX to multiple vehicles should be possible since PLANEX supports non-linear, parallel planning.

\section{Acknowledgments}

This work constituted a portion of research funded by the National Science Foundation under Grant DMC-86-15072 for Research on Cognitive Excavation Automation.

\section{References}

[Apte 89]

Apte,S., Object-oriented Implementation of a Domain Specific Planner for a Robotic Excavator, unpublished Master's Thesis, Dept. of Civil Engineering, Carnegie Mellon University, April 1989.

[Bullock 88] Bullock, D.M., Supervisory Control for Cognitive Excavation, unpublished Master's Thesis, Dept. of Civil Engineering, Carnegie Mellon University, August 1988.

[Bullock 89] Bullock, D.M. and I.J. Oppenheim, A Laboratory Study of Force-Cognitive Excavation, Technical Report, Dept. of Civil Engineering, Carnegie Mellon University, March 1989.

[Hendrickson 89] Hendrickson, C. and T. Au, Project Management for Construction, Prentice-Hall Ltd., 1989.

[Romero 88] Romero-Lois, H.R. and C. Hendrickson, A Strategic Planning and Monitoring System for a Machine Excavation Process, Technical Report R-88-175, Dept. of Civil Engineering, Carnegie Mellon University, Pittsburgh, PA, September 1988.

[Tatsuro 88] Tatsuro, M., "An Optimal Operation of a Bulldozer Running on a Weak Terrain," Proc. of the Fifth International Syumposium on Robotics in Construction, ISARC, Tokyo, Japan, June, 1988.

[Whittaker 85] Whittaker, W., "Cognitive Robots for Construction," Annual Research Review, Pittsburgh, PA, June, 1985.

[Zozaya 88] Zozaya-Gorostiza, C.A., Hendrickson, C., Rehak, D., and Lim, P.S., "CONSTRUCTION PLANEX: A Knowledge Intensive Planner for Construction Projects," Proc. Fifth International Symposium on Robotics in Construction, Japanese Robotics Association, June, 1988.

[Zozaya 89] Zozaya-Gorostiza, C., C. Hendrickson and D. Rehak, Knowedge-Based Project Planning for Construction and Manufacturing, Academic Press, Cambridge, MA, 1989. 